\title{
A global surveillance system for crop diseases
}

\author{
Global preparedness minimizes the risk to food supplies. \\ By M. Carvajal-Yepes, ${ }^{1}$ K. Cardwell, ${ }^{2}$ A. Nelson, ${ }^{3}$ K. Garrett, ${ }^{4}$ B. Giovani, ${ }^{5}$ D.G.O. Saunders, ${ }^{6}$ S. Kamoun, ${ }^{7}$ J. Legg, ${ }^{8}$ V. Verdier, ${ }^{9}$ J. Lessel, ${ }^{10}$ R.A. \\ Neher, ${ }^{11}$ R. Day, ${ }^{12}$ P. Pardey, ${ }^{13}$ M.L. Gullino, ${ }^{14}$ A.R. Records $*,{ }^{15^{*}}$ B. Bextine, ${ }^{16}$ J.E. Leach, ${ }^{17}$ S. Staiger,${ }^{1}$ J. Tohme ${ }^{1}$
}

Health Organization (WHO) and the U.S. CenTo satisfy growing demand for food, global agricultural production must increase by $70 \%$ by 2050 . However, pests and crop diseases put global food supplies at risk. Worldwide, yield losses caused by pests and diseases are estimated to average $21.5 \%$ in wheat, $30.0 \%$ in rice, $22.6 \%$ in maize, $17.2 \%$ in potato, and $21.4 \%$ in soybean [1]; these crops account for half of the global human calorie intake [2]. Climate change and global trade drive the distribution, host range, and impact of plant diseases [3], many of which can spread or re-emerge after having been under control [4] (see photo). Though many national and regional plant protection organizations (NPPOs and RPPOs) work to monitor and contain crop disease outbreaks, many countries, particularly low-income countries (LICs) do not efficiently exchange information, delaying coordinated responses to prevent disease establishment and spread. To improve responses to unexpected crop disease spread, we propose a Global Surveillance System (GSS) that will extend and adapt established biosecurity practices and networking facilities into LICs, enabling countries and regions to quickly respond to emerging disease outbreaks to stabilize food supplies, enhancing global food protection.

Global networks have improved human health, expediting global responses to hu-

\footnotetext{
International Center of Tropical Agriculture, Cali Colombia. "Oklahoma State University, Stillwater, $\mathrm{OK}$, USA. Department of Natural Resources, Faculty of GeoInformation Science and Earth Observation (ITC), University of Twente, Enschede, The Netherlands. ${ }^{\prime}$ University of Florida, Gainesville, FL, USA. ${ }^{\circ}$ European and Mediterranean Plant Protection Organization/Euphresco, Paris, France. 'John Innes Centre, Norwich, UK. 'The Sainsbury Laboratory, University of East Anglia, Norwich, UK. 'International Institute of Tropical Agriculture, Dar es Salaam, Tanzania. "French National Research Institute for Sustainable Development (IRD), CIRAD, University of Montpellier, Interactions Plantes Microorganismes Environnement, IPME, Montpellier, France. ${ }^{\circ 0} \mathrm{Gro}$ Intelligence, New York, NY, USA. "University of Basel, Basel, Switzerland. ${ }^{12}$ Centre for Agriculture and Biosciences International, Wallingford, UK. ${ }^{13}$ University of Minnesota, Minneapolis, MN, USA. "Torino University, Torino, Italy. "United States Agency for International Development, Washington, DC, USA. "University of Texas, Austin, TX, USA. "Colorado State University, Fort Collins, CO, USA.

Email:m.carvajal@cgiar.org,j.tohme@cgiar.org
}

man infectious disease outbreaks. The World ter for Disease Control and Prevention addressed challenging public health problems more effectively and rapidly by developing and maintaining surveillance systems with well-established network labs for diagnosis and promoting norms for sharing data and information during outbreaks [14]. In a similar spirit, with the United Nations General Assembly having proclaimed 2020 as the International Year of Plant Health to increase awareness among the public and policymakers about the importance of plant health [15], we foresee tremendous opportunity for a GSS to help governments deliver targeted and more cost-effective responses to plant disease outbreaks.

The International Plant Protection Convention (IPPC), adopted in 1951, provides the basis for collaboration by participating countries in NPPOs and RPPOs to improve the awareness of threats to agriculture from the entry and spread of regulated pests and pathogens. This system of 183 NPPOs and 10 RPPOs, in cooperation with the IPPC Secretariat and Commission for Phytosanitary Measures, faces multiple challenges, including the focus on a high number of regulated pests (352 in Europe alone), with limited human and financial resources.

Two types of infrastructure currently define a country's capacity for crop disease surveillance: specific/targeted and general/passive [7]. Specific and targeted surveillance infrastructure consists of labs at entry and trade points, customs and border patrol, seed inspection, and phytosanitary services, and includes coordinated agricultural pest surveys designed to prevent the introduction and movement of specific pests and diseases. Most IPPC and NPPO policy efforts are tied to targeted surveillance, which requires trained personnel to recognize regulated pests and pathogens and to establish an area as "free from" a given pest or disease for trade and quarantine purposes [7]. Despite the substantial global targeted surveillance infrastructure, only an estimated $2-6 \%$ of all cargo entering a country can be effectively biological invasive species through official entry points is barely constrained [8].

General or passive surveillance is aimed at detecting and diagnosing all pests and screened; thus, actual movement of potential crop diseases, not just those that are regulated. Passive surveillance personnel either spot diseases during field surveys or receive samples brought to labs distributed throughout a country or region. These are almost always the first detectors when an outbreak is occurring, and are often loosely networked groups of citizens; scientists and trained agronomists; university plant pathology labs; fee-for-service clinics supporting grower industries; CGIAR plant pathology labs; national networked labs, such as the U.S. National Plant Diagnostic Network (NPDN); national extension service personnel; private crop consultants; and pesticide salespeople and applicators.

For this infrastructure to be effective, connections between first detectors and downstream responders must be well coordinated. But, diagnosis capacity, information sharing and communications protocols are lacking or weakly established in most regions. Our reflection on many disease outbreaks is that whether in HIC or LIC, the passive surveillance infrastructure has the most in-field monitoring and trained eyes, but the least coordination from local to global. This is the sector we propose to network for the GSS, particularly including LIC, where risk assessment, diagnostic capacities, data sharing, and communication protocols need to be strengthened.

\section{COMPONENTS OF A GSS}

The model for the GSS draws on lessons learned from previous outbreaks, established and coordinated regional plant protection efforts and from the best practice implemented in HICs, such as the EPPO, the U.S. NPDN, the EU Reference Labs, and the Global influenza surveillance and response system (GISRS) coordinated by the WHO [5] [6].

Reviewing previous disease outbreaks helped to identify weakness that need to be strengthened and, strengths that could be leveraged in other regions.. One example is the recent cassava mosaic disease (CMD) outbreak in Southeast Asia. CMD is a viral disease that causes substantial yield loss in Africa, India and Sri Lankan and is an important impediment to trade in vegetativelypropagated planting material. A university plant pathologist made the first report of a 
new occurrence in Cambodia in 2015, alt2 hough these results were published in a sci3 entific journal until May 2016. Concerns 4 within the region about the economic impli5 cations of recognizing the presence of the 6 disease led to further delays in issue a cas7 sava warning, allowing CMD to spread into 8 neighboring regions and countries. Key con- from Europe, Brazil, and the United States and fed by a collaboration to develop an international IPPC Standard for the diagnosis of Xylella fastidiosa.

The GSS would comprise existing surveillance systems worldwide, but with a deliberate coordination of people, compilation and analysis of disease diagnostic data patterns, and a forward-looking goal of improved risk management at a global scale. It would create linkages between general and specific surveillance entities across countries to increase coordination in high-consequence disease detection, allowing optimization of early response and control. It would function through five interconnected networks: (i) diagnostic labs, (ii) risk assessment modeling teams, (iii) data standardization and management specialists, (iv) regular expert communications, and (v) a distributed operations management system, all sharing a cross-cutting capacity-development component. A pilot phase would focus on high-risk diseases causing high economic impact, in some of the world's most important crops (maize, potato, cassava, rice, beans, and wheat) in LIC. This would strengthen the capacity and link critical components of existing networks to better respond to high-risk diseases.

In the past decade, major advances have been made in disease diagnostics, especially through genome sequencing technologies; CRISPR-based diagnostics; bioinformatics tools for genomic epidemiology, genomic prediction, data mining, data analysis, and modeling; and expansion of social media platforms for information sharing [11]. Such advances will revolutionize the speed, accuracy, and wealth of information collected during disease outbreaks. One recent example is a near real-time, genomics-based, point-of-care diagnostics platform for wheat yellow rust, Mobile And Real-time PLant disEase (MARPLE) diagnostics, which has been integrated into an existing wheat rust early warning system in Ethiopia to directly inform disease risk forecasting (see photo). However, the benefits of faster, more accurate detection and diagnostic technologies have not been evenly applied to LIC, where emerging diseases can be particularly devastating. Concurrent efforts to leverage and deploy emerging technologies for disease monitoring and management in LIC must occur to effectively reduce the impact of crop diseases locally and disease spread globally.

The diagnostic laboratory network would promote standard protocols including those already proposed by IPPC, with ad- vanced techniques for faster and more accurate results and standardized information management and reporting. It would be coordinated by "regional hubs" that support the "spoke" diagnostic labs in a region, focusing specifically on diagnostic labs and extension services in LIC, in a similar approach as the U.S. NPDN with its five regional diagnostic networks. Most LIC plant protection services are under-resourced, so the GSS would strengthen labs, through training, regional connectivity, and resources. The regional hubs would link and support current NPPOs capacity and infrastructure for lab diagnostics and field-based surveillance with extension agents, plant breeders, and remote sensing-based platforms. The regional hubs would work with spoke teams to identify high-risk crops and pathogens based on local priorities. Regional hubs in collaboration with RPPOs and the IPPC would work to facilitate consensus with regional partners to update and coordinate existing standard operating procedures for diagnostics, sampling methods, and surveillance approaches. These capabilities would be integrated with the Risk assessment and Communication networks to support early field-based detection, diagnostic confirmation, and timely reporting in each region.

The current standards for pest risk analysis for quarantine pests were established by the IPPC in 2001. Using these standards as guidance, the risk assessment network would support the collection, integration, and management of risk- related data to develop analytical modeling and visualization tools, and for interpreting and communicating (via the GSS Communication and Data management networks) to key stakeholders through emergency alerts, regular bulletins, and updated priority lists for crop diseases. This network would recommend sampling strategies, supply updated risk estimates to the Diagnostic laboratory network, and would contribute to capacity development along with NPPO, universities, government personnel, and private groups.

The Data management network would develop consensus with participating countries on data collection standards and access protocols to support the collection, curation, storage, analysis, and management of plant disease data. This would be based on rules of data access and use, such as in public health emergencies and the Pandemic Influenza Preparedness (PIP) Framework from the WHO, which shares genetic sequence data in a rapid, timely, and systematic manner from the originating lab and among WHO GISRS labs. The GSS would provide data to inform 
the Risk assessment and Communication networks to guide timely responses.

The GSS would incentivize data sharing (including open-source data) by deploying FAIR (findable, accessible, interoperable, and reusable) data principles [12] although accessibility outside the system would be decided on a per country or regional basis. Although individual countries might want to limit public availability to sensitive crop disease data, and threats to sharing data have been reported [13], the GSS would ensure that norms for sharing information and data usage are established. The GSS, through this network, would work with the plant pathology community and seek support from plant pathology journals, similar to the agreement and support from the International Committee of Medical Journal Editors (ICMJE), by not prejudicing journal publication because of pre-publication dissemination of information that is critical to public health emergencies, as when declared by WHO. A code of ethics for plant health emergencies by The International Society for Plant Pathology (ISPP) is under discussion. The benefits of coordinated efforts to share pathogen-associated data, in the case of the outbreak of wheat blast in Bangladesh, allowed identifying the most likely origin guiding the decisions and efforts.

The Communication network would facilitate dialogue across all networks, internally to the system and externally to participating host governments, for raising awareness and coordinating timely responses to disease outbreaks. This network would expedite the transfer of knowledge derived from the Diagnostic and Risk assessment networks by identifying the most appropriate source and ensuring the timely, responsible, and secure transfer of knowledge.

Key members of international plant protection organizations; partner networks such as NPDN, IPPC, and RPPOs; and CGIAR liaisons would oversee the global management of regional operations. This operational management network would provide governance for an integrated surveillance system to promote global awareness of and preparedness for crop disease outbreaks. Its main activities would be operating the networks, coordinating partners, administrating budgets, fundraising, and establishing policies and guidelines.Each network would include capacity development, ensuring that local and regional institutions increase their capacity at three different levels by training individuals to increase skills and knowledge; providing resources, services, and infor- mation to strengthen organizations; and facilitating institutional cooperation and collaboration.

\section{IMPACT AND IMPLEMENTATION}

The GSS would detect threats and risks to global food supplies and support timely response. Countries and regions will benefit by increasing their capacity to predict, detect, communicate, and effectively respond to emerging crop disease outbreaks. This will be possible by leveraging different lessons learned from existing national and regional plant protection systems, such as NPDN established in 2003 [5] or EPPO created in 1951. The proposed GSS would need to tackle challenges such as enhancing awareness with each country's Ministry of Agriculture, and among RPPO and policymakers about the GSS and the function of the regional hubs; and establishing an integrated governance approach with long-term buy-in and sustainable funding.

In the IPPC development agenda for 2020-2030, IPPC and FAO highlighted the need to strengthen surveillance systems, with diagnostic laboratory networks as a key component. We encourage the annual G20 Agriculture Ministers Meeting, the World Bank Group, and FAO, among others, to join efforts toward enhancing cooperation for a multi-year action plan for the proposed GSS to more effectively reduce the impact of crop diseases and increase global food security.

REFERENCES AND NOTES

1. S. Savary, et al., Nat. Ecol. Evol., 3, 430439,(2019).

2. FAOSTAT, "Food and Agriculture Organization ofthe United Nations," FAOSTAT Statistics Database, 2018. [Online]. Available: http://www.fao.org/faostat/en/. 3. Y. Elad and I. Pertot, J. Crop Improv., 28, 99-139, (2014).

4. S. Bhattacharya, Nature, 542, 145-146,(2017).

5. J. P. Stack, et al., Plant Dis., 98, 708-715, (2014).

6. A. S. Monto, Influenza Other Respi. Viruses, 12, 10-12, (2018).

7. (IPPC) Secretariat of the International Plant Protection Convention, "ISPM 6 Surveillance," 2018, no. ISPM 06, p. 14.

8. T. T. Work, et al., “Arr Biol. Invasions, 7, 323-332, (2005).

9. M. T. Islam et al., BMC Biol., 14, 84, (2016).

10. B. Giovani, et al.,https://popups.uliege.be:443/17804507, (2019).

11. A. Hubbard et al., Genome Biol., 16, 23, (2015).

12. M. D. Wilkinson et al., Sci. Data, 3, 160018, (2016).

13. C. dos S. et al., Science, 362,. 404-406, (2018).

14. K. Modjarrad, et al., PLOS Med., 13, e1001935, (2016).

15. "Global Initiative Announced to Protect World's Plants from Pests - SeedWorld." [Online] Available: https://seedworld.com/globalinitiative-announced-to-protect-worldsplants-from-pests/. [Accessed: 28-Jan-
2019].

\section{ACKNOWLEDGMENTS}

The authors acknowledge support from the Rockefeller Foundation, Gatsby charitable Foundation, BBSRC, BASF Plant Science, GIZ and thank Adriana G. Moreira from the IPPC Secretariat and FAO/UN for her thoughtful comments and feedback. The views and opinions in this paper are the product of a group discussion convened at the Bellagio Center Conference Program granted by the Rockefeller Foundation and the Institute of International Education (IIE). The views and opinions expressed in this paper are those of the authors and not necessarily the views and opinions of the United States Agency for International Development.

10.1126/science.aaw1572

\section{FIGURE LEGENDS}

Figure 1. Recent disease outbreaks affecting farmers in LIC as well as in Europe: (a) stem rust symptoms on durum wheat in Sicily, Italy (2017, by Biagio Randazzo); (b) cassava mosaic disease symptoms in Cambodia (2016); (c) wheat blast disease symptoms spotted in Bangladesh (2016, by Tofazzal Islam); (d) capacity building in emerging genomic-based surveillance techniques through deployment of Mobile And Realtime PLant disEase (MARPLE) diagnostics in Ethiopia (by Matt Heaton, John Innes Centre). 